\title{
Can Tsunami Waves in the South China Sea Be Modeled with Linear Theory?
}

\author{
Yingchun Liu ${ }^{1,2}$, Yaolin $\mathrm{Shi}^{2}$, Hailing Liu ${ }^{1}$, \\ Shuo M. Wang ${ }^{4}$, David A. Yuen ${ }^{3,4}$, and Hui Lin Xing ${ }^{5}$ \\ ${ }^{1}$ South China Sea Institute of Oceanology, Chinese Academy of Sciences, Guangzhou, China \\ ${ }^{2}$ Graduate University of Chinese Academy of Sciences, Beijing, China \\ spring.yingch@gmail.com, shiyl@gucas.ac.cn \\ ${ }^{3}$ Minnesota Supercomputing Institute, University of Minnesota, Minneapolis, USA \\ ${ }^{4}$ Department of Geology and Geophysics, University of Minnesota, Minneapolis, USA \\ ${ }^{5}$ ESSCC, University of Queensland, Australia
}

\begin{abstract}
We have compared the results from linear and nonlinear theories of the shallow-water equations applied to the South China Sea. Our results indicate that tsunami waves in the South China Sea can be modeled with linear theory. There is little difference in the probability predicted by nonlinear theory and that forecasted by linear treatment on tall waves, more than two meters high, which may impinge on Hong Kong, Macau and Taiwan. This probability is estimated to be $10 \%$ in the next century.
\end{abstract}

Keywords: Tsunami, Shallow-water equation, Numerical computation, South China Sea.

\section{Introduction}

The recent Pingtung earthquake occurred on December 26, 2006 in southern Taiwan has called our attention to possible tsunami hazards along the Chinese coast from large earthquakes from Taiwan in the north to the Manila trench in the south. Numerical simulation based on linear theory with the shallow-water equations is a good candidate for timely prediction due to much smaller amount of computation involved. The South China Sea (SCS) (Fig. 1), together with Taiwan to its east and the Philippine arc-trench, form complicated channel-basin structural system. The majority of earthquakes in South China Sea region are distributed around South Taiwan and Philippine (Fig.11), especially along the Manila trench fault, which is the region most likely to generate tsunamis in the South China Sea. Historical seismic tsunami catalog shows that tsunamis have occurred close to Taiwan and Manila trench.

\section{Comparison of Linear and Non-linear Modeling}

The initial condition of the linear shallow water equation is computed according to Okada's work [3], which numerically predicts the water level changes due to earthquake faulting. Rectangular 2-D fault and half-space elastic models are adopted to represent major seismic faults for calculating the earthquake induced tsunamis. The earthquake 


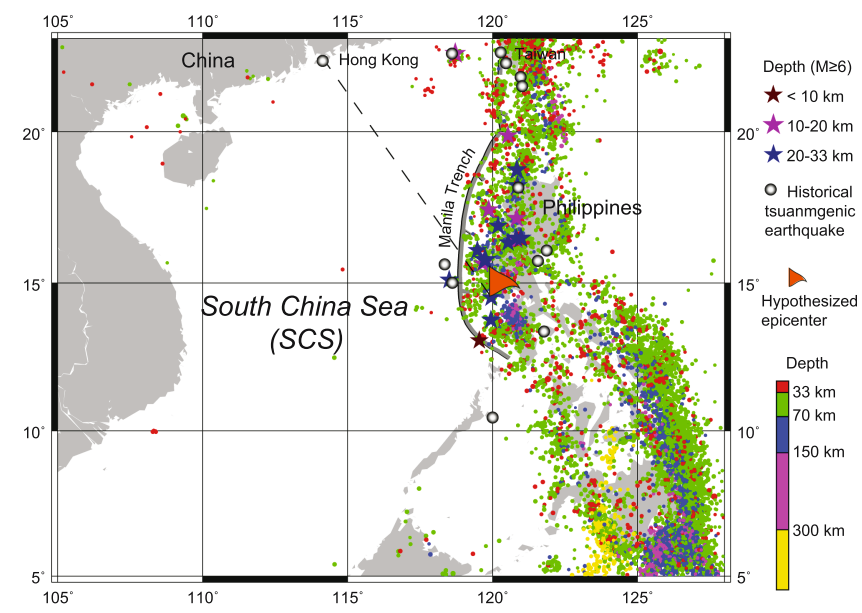

Fig. 1. Earthquake distribution with the epicenter depth in South China Sea and its adjacent regions. Database comes from NEIC. Major shallow earthquakes with magnitude over 6 are marked with star symbol. Grey balls represent the historical seismic tsunamis catalog from NGDC/NOAA. The marked hypothesized epicenter is used in Fig.3 and 4. A total of 31 receivers are placed on a straight line from epicenter to Hong Kong.

magnitude is set to be 8.0. In the rupture models, source parameters (rupture length $L$, width $W$, and the average slip $D$ ) are derived from theoretical and empirical relationships [5]. The fault dips and strikes from the composite fault plane solutions come from the average dip of the fault segments according to the Harvard catalog. The following linear shallow-water equations (11) are employed:

$$
\frac{\partial \eta}{\partial t}+\frac{\partial M}{\partial x}+\frac{\partial N}{\partial y}=0 ; \frac{\partial M}{\partial t}+g D \frac{\partial \eta}{\partial x}=0 ; \frac{\partial N}{\partial t}+g D \frac{\partial \eta}{\partial y}=0 .
$$

Due to the existence of the shallow water regions, for comparison, we also apply the non-linear shallow-water model. Here we include the effect of the friction coefficient on the wave height. The non-linear equations (2) are given by:

$$
\begin{aligned}
& \frac{\partial \eta}{\partial t}+\frac{\partial M}{\partial x}+\frac{\partial N}{\partial y}=0 \\
& \frac{\partial M}{\partial t}+\frac{\partial}{\partial x}\left(\frac{M^{2}}{D}\right)+\frac{\partial}{\partial y}\left(\frac{M N}{D}\right)+g D \frac{\partial \eta}{\partial x}+\frac{n^{2}}{D^{7 / 3}} M \sqrt{M^{2}+N^{2}}=0 \\
& \frac{\partial N}{\partial t}+\frac{\partial}{\partial x}\left(\frac{M N}{D}\right)+\frac{\partial}{\partial y}\left(\frac{N^{2}}{D}\right)+g D \frac{\partial \eta}{\partial y}+\frac{n^{2}}{D^{7 / 3}} N \sqrt{M^{2}+N^{2}}=0 .
\end{aligned}
$$

In both models where $\eta$ is the water height, $t$ is time, $x$ and $y$ are the horizontal coordinates, $M$ and $N$ are the discharge fluxes in the horizontal plane along $x$ and $y$ coordinates, $h(x, y)$ is the undisturbed basin depth, $D=h(x, y)+\eta$ is the total water depth, $g$ is the gravity acceleration, and $n$ is the Manning roughness.

We have employed in our simulations the linear tsunami propagation model TunamiN1 and nonlinear model Tunami-N2, that were developed in Tohoku University (Japan) and provided through the Tsunami Inundation Modeling Exchange (Time) program [1]. These models solve the governing equations by the finite-difference technique with a leap-frog scheme [1]. We use open boundary condition in these models, which permits 

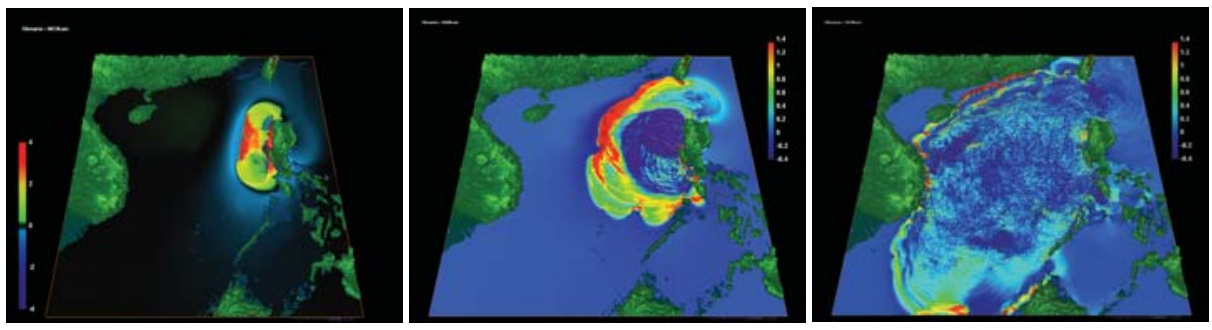

Fig. 2. Visualization of the tsunami wave propagation at three different times: 12 minutes, 72 minutes, and 479 minutes. Four meters represent the maximum height shown.

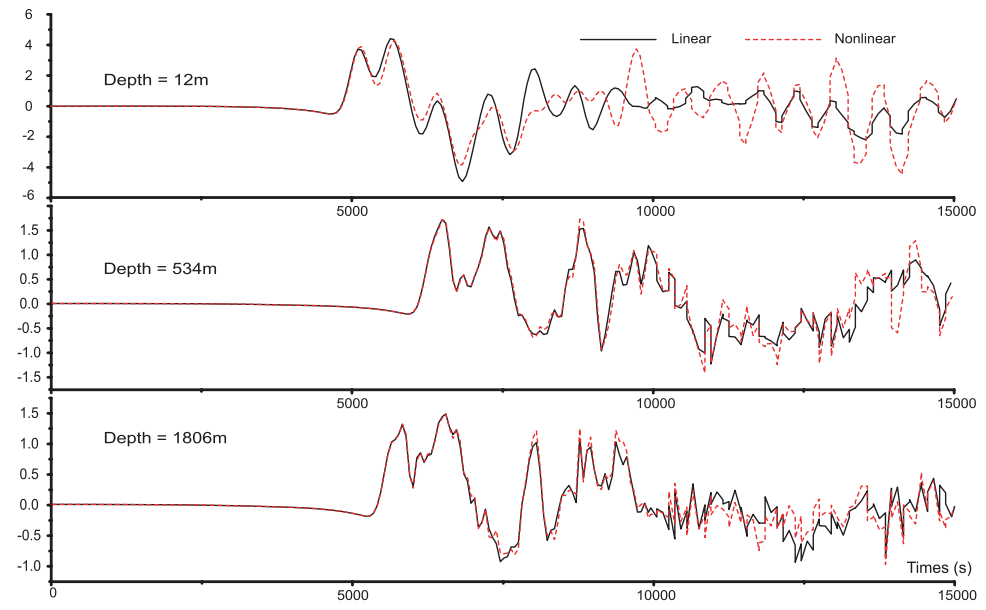

Fig. 3. Comparison of water heights in time histories with linear and nonlinear models in various water depths: $12.0 \mathrm{~m}, 534.0 \mathrm{~m}, 1806.0 \mathrm{~m}$. These waves are taken at the receivers located in $\mathrm{s}$ straight line between the epicenter (marked in Fig.1) and Hong Kong.

free outward passage of the wave at the open sea boundaries. The bathymetry of the South China Sea was obtained from global seafloor topography (Etopo2) with grid resolution of nearly $3.7 \mathrm{~km}$. The total number of grid points in the computational domain is 361,201 , which is $601 \times 601$ points. The time steps in both models are selected to be 1 second to satisfy the temporal stability condition.

We have visualized in Fig. 2 one set of simulated tsunami wave propagations with the nonlinear model. The duration of wave propagation is 6.0 hours. This hypothesized seismic tsunami occurs southwest to Philippines $\left(14.5^{\circ} \mathrm{N}, 119.2^{\circ} \mathrm{E}\right)$, with a magnitude of 8.0. For comparison between the linear and nonlinear models, we perform our simulations under the condition of $n=0.025$ as the bottom friction coefficient in this application [1]. Tsunamis occurring at this location are furthest to the coast of mainland China. Due to the longest propagation time and very strong wave energy of this tsunami, 
strong oscillations, reflection, and interference characteristics of tsunami waves can be well observed near the islands.

To further validate the wave propagation process in linear and nonlinear models, we place 31 receivers along the straight line between the epicenter in the Philippines and Hong Kong, as shown in Fig. 1 It has been found in our simulation that, there is one critical region with depth between 400 and 500 meters. The nonlinear character dominates the wave with small wavelengths. Above this depth, both linear and nonlinear models generate similar wave shapes and wave magnitudes. In other words, with the ratio of wave height to water depth smaller than 0.01 , wave propagation can be modeled by linear theory with reasonable accuracy. We select three receivers (Fig.3) at different water depths.

\section{Probabilities of Potential Tsunami Hazard of SCS}

In South China Sea, over three-fourth of this area is deeper than 500 meters. Due to dominance of the deep region, the linear model is expected to perform well. Here we will compute the probabilities of the tsunami hazard in the South China Sea area by using both linear and non-linear models. In the nonlinear model, the Manning roughness is 0.025 for the natural channels in good condition which is suitable for the South China Sea. The probability of seismic studies for the South China Sea and the adjacent areas was based on the Gutenberg-Richter (G-R) relationship [2]. The number of earthquakes is computed from the probability of seismic evaluation [6]. Earthquakes are sampled with the epicenter randomized according to a modified G-R distribution [4]. To estimate precisely the probability of tsunami hazard, 13 seismic tsunami models with magnitudes ranging from 6.5 to 8.0 are computed. We record the maximum positive amplitudes of the tsunami over a sufficiently long period of time.

Our probability for tsunami wave occurrence is computed from the probability of earthquake occurrence and the probability of maximum wave height of all seismic tsunami induced by such earthquakes. The tsunami wave with heights of [1.0m, 2.0m] and heights over 2.0 meters are considered for the hazard prediction. We forecast that the probability for tsunami wave with more than 2.0 meters to hit within the next 100 years is $10.12 \%$ for Hong Kong and Macau, 3.40\% for Kaoxiong, and $13.34 \%$ for Shantou with the linear model. With the nonlinear model, the probability is the same for the same tsunami wave height at Hong Kong and Macau, and Kaoxiong, while a lower probability at Shantou of $10.12 \%$ is found. In general, the probabilities for most coastal cities do not change with the usage of nonlinear theory.

\section{Acknowledgement}

We would like to thank Professor Fumihiko Imamura for his providing the code of TUNAMI_N1, TUNAMI_N2, and his kind guidance on the tsunami numerical method. This research is supported by National Science Foundation of China (NSFC-40574021, 40676039) and the EAR program of the U.S. National Science Foundation. 


\section{References}

1. Goto, C., Ogawa, Y., Shuto, N., and Imamura, N.: Numerical method of tsunami simulation with the leap-frog scheme (IUGG/IOC Time Project), IOC Manual, UNESCO, No. 35 (1997)

2. Gutenberg, B. and Richter, C. F.: Seismicity of the Earth and Associated Phenomena, Princeton Univ. Press, Princeton (1949)

3. Okada, Y.: Surface deformation due to shear and tensile faults in a half-space, Bull. Seism. Soc. America 75 (1985) 1135-1154

4. Reiter, L.: Earthquake Hazard Analysis: Issues and Insights, Columbia Univ. Press, New York (1990)

5. Wells, D., Coppersmith, K.: New empirical relationships among magnitude, rupture length, rupture area, and surface displacement. Bull. Seismol. Soc. Am. 84 (1994) 974-1002

6. Liu, Yingchun, et al.: Tsunami Hazards From Potential Earthquakes along South China Coast. submitted to Physics of the Earth and Planetary Interiors (PEPI), Elsevier, 2007 